\title{
AUTOMATED PROCESS CONTROL SYSTEM OF MOBILE CRUSHING AND SCREENING PLANT
}

\author{
Andrey Ostroukh ${ }^{1 *}$, Nataliya Surkova ${ }^{1}$, Oleg Varlamov ${ }^{1,2,3}$, Valery Chernenky ${ }^{3}$, Alexander Baldin ${ }^{3}$ \\ ${ }^{1}$ Moscow Automobile and Road State Technical University (MADI), Moscow, Russian Federation \\ ${ }^{2}$ RI MIVAR, Moscow, Russian Federation \\ ${ }^{3}$ Bauman Moscow State Technical University (BMSTU), Moscow, Russian Federation
}

The article research the problem of automating the mobile crushing and screening plant as a complex multi-level system. The analysis of the characteristics of the technological equipment and individual aggregates of the mobile crushing and screening plant is performed.A software for automated cone crusher control system, that allows to integrate a set of interconnected technological units in the control of one hardware, controller and human-machine interface on the touch panel in the operator station.Proposed human-machine interface based on a set of technical, informational, mathematical and software tools for controlling the mobile cone crusher, which ensures efficient operation of the technological control object as a whole.In accordance with the proposed concept, a complex automated system should provide an optimal level of automation for information collection and processing to form control signals and transfer them without loss and distortion to actuators in order to achieve efficient operation of the technological line for the crushing and screening plant.

Key words: Automation processes, Automated process control system (APCS), Crushing and screening plant, Jaw crusher, Cone crusher, Mnemonic scheme, Human-Machine Interface (HMI)

\section{INTRODUCTION}

Modern crushing and screening production of stone materials is a complicated technological complex with a set of operations including the delivery of raw material, its purification and pre-sorting, crushing during several stages including sorting the required commodity fractions into the stages of crushing, storing and transporting to the final consumer. Crushing and screening technological processes are among of the most important processes in construction materials formulation being, on the contrary, not highly effective.

In order to increase crushing and screening processing efficiency of stone materials one needs to develop new multi-level automatic process control systems of crushing and screening plants; in the last few years these plants become more and more transportable, e.g. movable from one site to another.

Modern automatic process control systems incorporate various elements brought together in order to reach some goal using branched interlocking bonds [1 - 6]. Taking into account the evolving modern concepts of "Internet of Things" and "Industry 4.0", the increasing number of industrial devices, machines and units are equipped with modern automation systems developed with the use of modern industrial controllers. Industrial devices, machines and aggregates have a permanent connection to the global network with used wireless communication channels. Control is performed with used Human-Machine Interface (HMI) implementing in software on interactive touch panels (touchscreens).

In the near future, machines (or rather their software) will make many of the traditional deterministic decisions that a human would otherwise make, and artificial intelligence applications will facilitate even more abstract decisions through machine learning and deep-learning algorithms. MIVAR computations as a scientific field began developing in the 80's years of the 20th century [17 - 19]. Developers faced a challenge of reducing the requirements for computational systems by using more sophisticated mathematical models. Three pillars of MIVAR technologies are innovative multi-dimensional databases, a unique MIVAR algorithm of information processing and a conceptual cognitive model of objects description and their relations.MIVAR-based technologies comprise the basis for evolutionary leap in robotic systems development: ranging from mechanical manipulators and simple mechanisms to reasoning systems and their social cooperation.

\section{MOBILE CRUSHING AND SCREENING PLANT UNITS}

Crushing and screening plant processing kit is a branched continuous handling system consisting, for instance, of clatters, crushing station and several ribbon conveyors; the more complicated version includes dozens of equipment units included into one technological process and functioning automatically [7 - 15].

The choice of the crushing and screening plant technological scheme depends on the type of raw materials being processed, its physical characteristics, quality requirements and the purpose of the finished product. In addition, the crushing and screening plant design project depends on the required ratio of cubed grain, the 
planned capacity, as well as the willingness of the crushing and screening plant owner to bear a certain share of the cost on restoration of the working capacity of its working bodies.

Research object is the Metso NW Rapid wheel-mounted transportable crushing and screening plant (Figure 1).

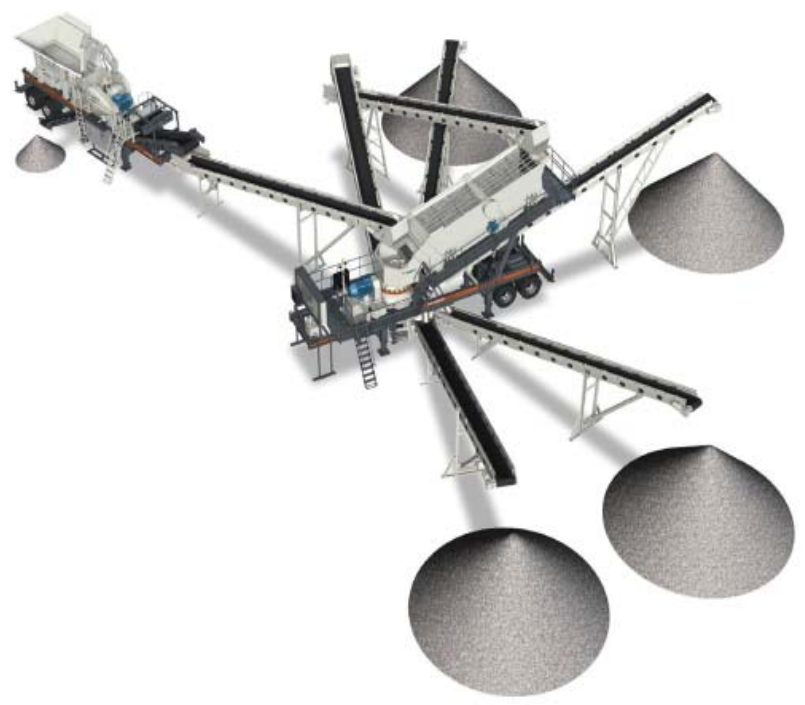

Figure 1: Metso NW Rapid wheel-mounted crushing and screening plant design project

The relative performance of a crushing and screening plant can be calculated by the following formula:

$$
Q_{c}=\sum_{i=1}^{N} Q_{0_{i}}=\frac{1}{\Theta(\rho)} \sum_{i=1}^{N} n_{i}
$$

where

$Q_{0 i}$ - relative productivity of all $n_{i}$ crushing aggregates of the $i$-th type;

$\theta(\rho)$ - performance loss function.

The function $\theta(\rho)$ determines the loss of productivity of the crushing and screening plant due to the downtime of the crushing equipment with the corresponding production $\rho=N / \mu$, estimated by the ratio of the average number of unoccupied crushers to their total number.

The expression for the performance loss function can be written as:

$\theta(\rho)=1+M_{p}$

Coefficient of idle crushing and screening plant:

$$
M_{p}=\frac{\sum_{k=1}^{n_{i}}\left(n_{i}-k\right) \pi_{k}(\rho)}{n_{i}}
$$

where

$M_{p}$ - average number of unoccupied crushers;

$\pi_{k}(\rho)$ - set of stationary probabilities of the system state; $n_{i}$ - number of crushers $i$-th type.

The task of optimizing the crushing and screening plant for maximum performance is written as:

$$
Q_{c}=\frac{1}{\Theta(\rho)} \sum_{i=1}^{N} Q_{0_{i}} \rightarrow \max
$$

Analysis of this expression shows that obtaining the maximum performance of the crushing and screening plant, the chosen configuration, is possible provided that the minimum $\theta(\rho)$.

Obviously, in order to obtain the maximum relative performance, the global minimum of the loss function, on the set of values of the production load $\rho$ should tend to unity. When solving engineering problems, efficiency evaluations are usually formulated in direct or in reverse formulation.

The main units of transportable crushing and screening plant which provide high quality of crushing products as well as high performance, is:

- Metso NW106 wheel-mounted transportable assembly (fig.2) equipped with Nordberg C106 roll-jaw crusher for primary crushing of stone products;

- Metso NW220GPD wheel-mounted transportable assembly equipped with Nordberg GP220 cone crusher (fig.3) and four-deck vibratory screen with double-angle of DS series for the final crushing of stone materials and their sorting into specified fractions.

\section{Mobile crushing and screening plant with jaw crusher}

Jaw crushers are used for primary and secondary crushing of solid and abrasive rocks (granite, diabase, sandstone). Crushing is performed by two 'jaws' - slabs, one of which perform oscillatory motions.

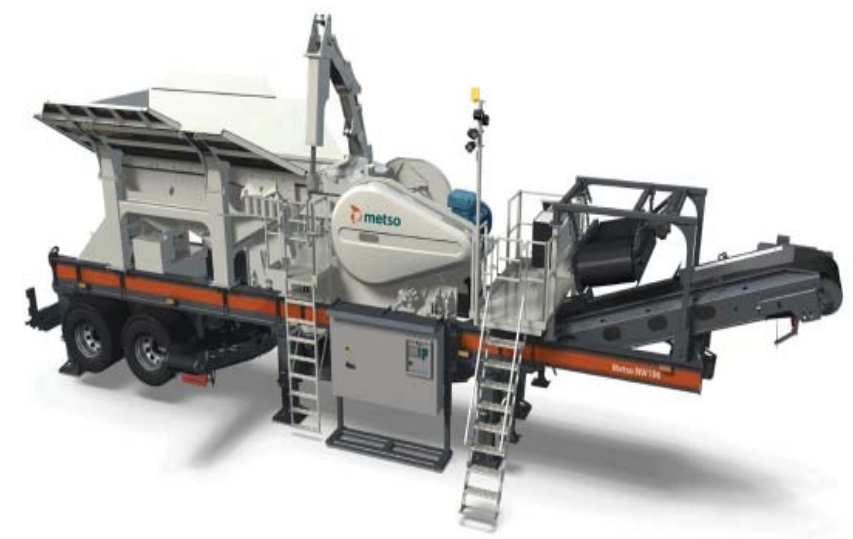

Figure 2: Metso NW106 mobile crushing and screening plant with Nordberg C106 jaw crusher 
The width of the receiving hole determines the maximum possible size of the pieces loaded into the crusher.

Pieces of the initial material being loaded move downwards by gravity and are crushed in jaws. Large initial pieces are crumbled and processed, down to sand and dust. Pieces which are smaller than the size of the exit slot $b^{z}$ come out of the crusher, and the material remaining between the cheeks in the grinding chamber continues to be processed. Due to the complexity of the drive structure, a simple swing of the jaw is replaced by a wave motion that facilitates the movement of the material along the crushing chamber towards the exit slit.

The technical performance of jaw crushers is determined by the formula:

$$
Q_{t}=60 \mathrm{Vn \mu}
$$

where

$V$ - is the volume of material falling out through the discharge gap per revolution of the eccentric shaft, $\mathrm{m}^{3}$;

$n=200 \ldots 250 \mathrm{~min}^{-1}$ - eccentric shaft rotational frequency (swing frequency of the movable jaw);

$\mu \approx 0,3 \ldots 0,65$ - coefficient incorporating the presence of voids between the stones being crushed.

The maximum size of the crushing product is determined by the formula:

$$
z_{\max }=K_{p} K_{g} \sqrt{\left(\frac{t}{2}\right)^{2}+\left(b^{z}+h\right)^{2}}
$$

where

$K_{p}$ - coefficient incorporating the configuration of the crushing jaw (triangular corrugations $K_{p}=0,8$; trapezoidal $\left.K_{p}=0,7\right)$;

$K_{g}$ - coefficient incorporating material properties (touchstone $K_{g}=1,1$; granite $K_{g}=1,0$ );

$t$ - step of corrugations, $\mathrm{mm}$ (for approximate calculations $\left.t=b^{2}\right)$;

$b^{2}$ - slit width, mm;

$h$ - height of corrugations, $\mathrm{mm}(h \approx t / 2)$;

The weighted average size of the crushed product (mean size) is determined by the following formula:

$z_{c B}=k_{d} b^{z}$,

where

$b^{z}$ - output slit width, $\mathrm{mm}$;

$k_{d}$ - weighted average coefficient, which is 0.65 for jaw crushers with a width of the receiving opening $B=600$ $\mathrm{mm}$ and less; with the width of the receiving hole of $\mathrm{B}=900 \mathrm{~mm}$ and more $k_{d}=0,8$.

\section{Mobile crushing and screening plant with cone crusher}

Cone crushers are the most effective for medium and fine crushing of strong and highly durable rocks. They carry out crushing continuously due to the rotation of the eccentric conical rotor - the "crushing cone" inside the outer stationary cone.

Cone crushers use continuous rotation instead of oscillations; unlike jaw crushers the change in clearance is made not simultaneously along the entire width of the gap, but alternately by its variation along the length. The working clearance in the cone crusher varies not simultaneously along the entire width as in the jaw crusher, but continuously along a circle, helping to improve the quality of crushing. The input and output gaps in the cone crusher are in the form of concentric rings. The maximum and minimum size of the output slot is set by the adjusting device.

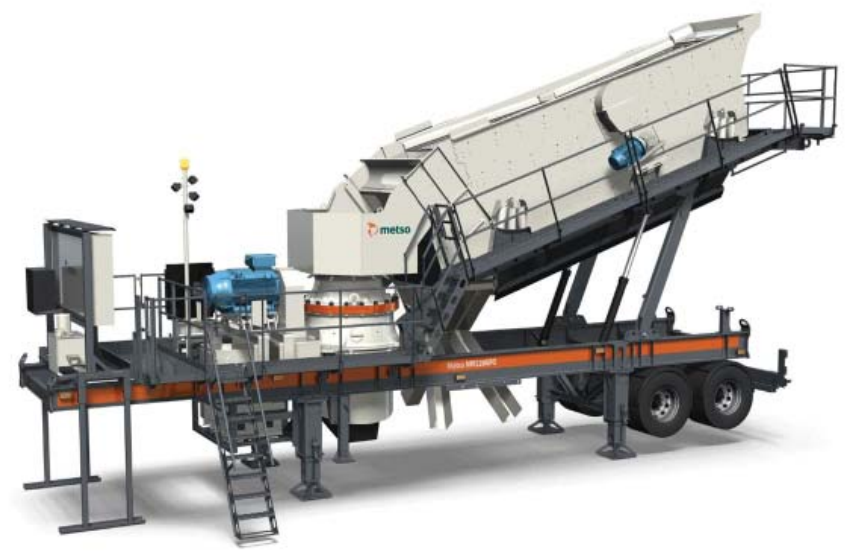

Figure 3: Metso NW220GPD mobile crushing and screening plantwith Nordberg GP220 cone crusher

The size of the cone crushers product depends mainly on the size of the output gap and the strength of the rock. The weighted average size of the crushing product zsv for soft, medium and strong rocks in fractions of the output gap bz of the cone crusher is determined by the formula:

$z_{c B}=K_{p} b^{z}$,

where $K_{p}=2,2 \div 2,8$ (for soft rocks), $K_{p}=2,6 \div 3,6$ (for intermediate rocks), $K_{p}=3,0 \div 5,0$ (for solid rocks).

The technical productivity of cone crushers is determined in $\mathrm{m} 3 / \mathrm{s}$ by material batch volume per one crushing cycle:

$Q_{t}=0,45 \pi n b^{2} / D$

where

$n$ - is the frequency of cone rotation, $c^{-1}, b^{2}$ is the width of the output slit, m;

$I$ - is the length of the parallel zone of the output gap, m; $D$ - is the diameter of the crushing cone base, $\mathrm{m}$. 


\section{THE POWER SUPPLY AND AUTOMATION SYSTEM}

The power supply and automation system (Figure 4) is built on a modular principle, which provides the possibility of manufacturing reconstruction or its modernization.

The control commands of the crusher drives, the size of the output gap, the feeding conveyor, the screen, the conveyor of the finished products are formed on the operator's automated workstation where the program is executed in accordance with the specified algorithm for optimal control of the crushing process.

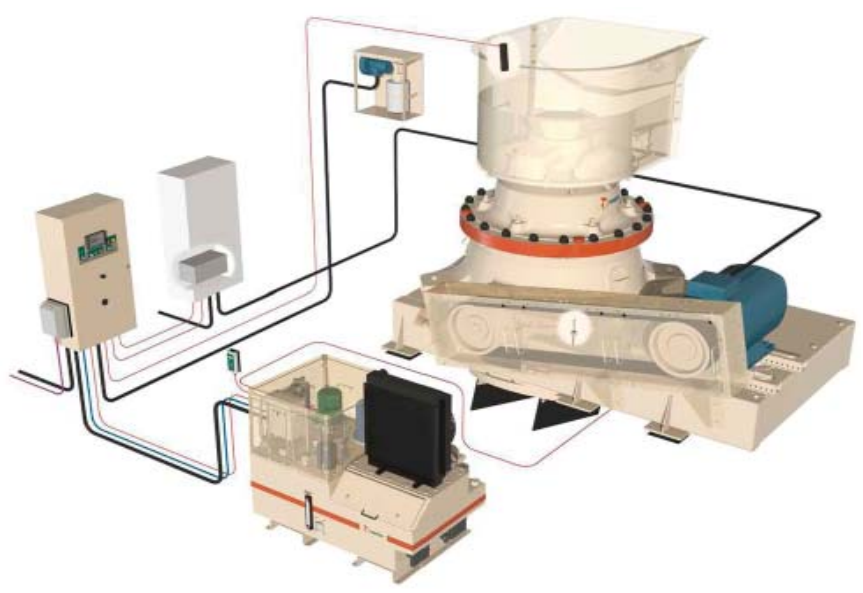

Figure 4: Nordberg GP220 Cone Crusher Power and Automation System

The local control during automation is provided by information channels with the necessary number of sensors controlling the relevant parameters.

In case of the crusher they are the sensors of the drive speed (frequency), the size of the output gap, the force (moment) of crushing and energy being consumed.

The process of crushing is controlled by changing the size of the output gap and the drive speed regardless of the type of crusher (by adjusting the speed of the crusher drive) [9 - 12].

Simultaneous control of the crusher output gap size and the speed of the drive can be defined as two-dimensional which achieve the required value of the crusher capacity along with ensuring the required fractional composition of the output product.

Controlling of the output gap size alone is the main way to obtain the output of the required fractional composition and it can be defined as one-dimensional. It is the one-dimensional way of controlling the process of fragmentation that is mainly the subject of research in this dissertation work. The usage of this control method does not impose any restrictions on the crusher performance, considering that the required fractional composition is the main indicator that ensures the crushing and screening plant effectiveness.

The choice of the method for controlling the crushing process also depends on the crusher design features, the chosen technological process, the conditions of the crushing and screening plant operation (planned production of crushing products or production under conditions of random demand).

Communication functions can be performed by wired communication with high reception and transmission interference immunity based on fiber optics, and in some cases also by wireless communication coupled with modern network industrial interfaces.

\section{TOUCH SCREEN AND CONE CRUSHER CONTROL PANEL}

Metso IC automated control system for crushing and sorting process of stone materials (fig. 5) is developed on the basis of the SCADA system [16] and provides a simple and safe use of the crushing plant. The system allows you to control the feeder, crusher and conveyors with a single touch screen.

Metso IC automation solutions are designed to meet the customer's needs and the requirements of the crushing plants for continuous productivity, safety and easy monitoring of the crusher's parameters. Customers can be assured of the proper crusher functioning and minimal downtime under all the operating conditions thanks to the optimized start, stop and workflow algorithms that form the basis of the Metso IC automation system.

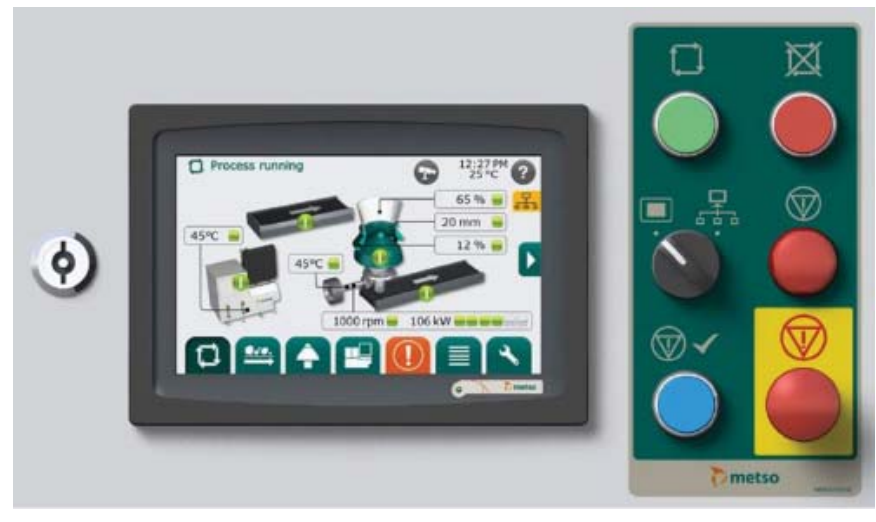

Figure 5: Touch screen and control panel of the Metso IC cone crusher

Metso IC automation system allows you to optimize the crushing equipment performance. This is achieved through clearly defined safety control parameters such as oil temperature and pressure and drive power, which are indicators of the actual working crusher load. The Metso IC automation system monitors the crusher's condition and identifies faults and problems in advance. Thus, it is possible to eliminate the problem without waiting for serious and costly consequences.

\section{AUTOMATION SYSTEM COMPONENT INTEGRATION}

The upper level of crushing and screening plant automation is the automated system of crushing and screening production technological preparation for automation of all the production control processes. 
The main task of the lower level automation is the organization of crushing process optimal control, the purpose of which is to provide the required fractional composition of the crushing product. The lower level automation task is the particular task of the general production automation task. At the same time, the lower level of crushing and screening plant automation can be considered as the automated control system for the technological process of crushing.

The Metso IC automation system can be easily connected to any general plant automated control system used for crushing and screening equipment (Figure 6).

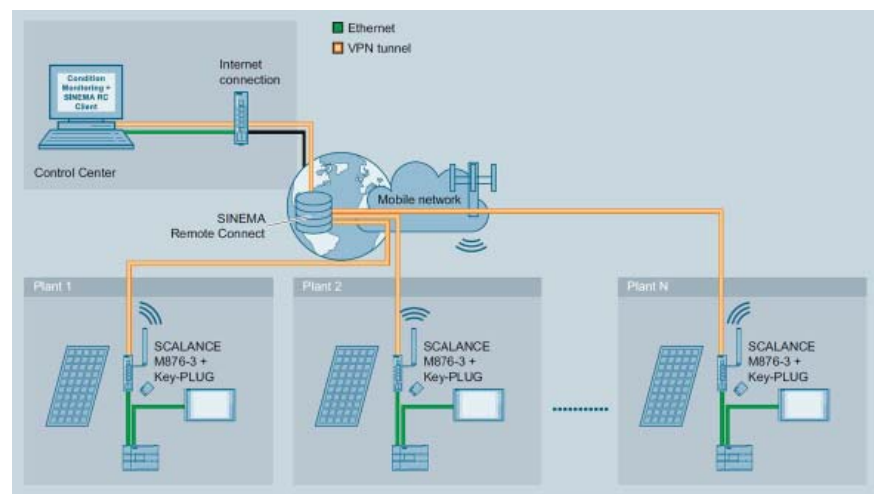

Figure 6: Automation system component integration

Such integration will provide the possibility of crusher centralized control as well as the whole enterprise.Also allow operator to safely monitor and regulate the crushing equipment operating parameters from one operator's console.

To increase the intelligence of the Automated Process Control System, the MIVAR technologies of creating logical artificial intelligence will be applied [16 - 19].

\section{CONCLUSION}

In this way, the features of the functioning of the crushing and screening plant scheme elements have been determined due to the need to automate the crushing and sorting production.

Based on the design parameters of the crushers it is shown that the size of the crushing product, which is a certain weight average diameter of the crushed product grain is its main stochastic characteristic.

It is established that the task of the crushing process automatic regulation is to maintain the final product's specified size and to maximize the energy supplied to the crushing aggregates by optimally loading the crushers.

The software for the automated process control system of crushing stone materials has been proposed, which makes it possible to integrate a set of interconnected technological units in the one hardware and technical complex management, the efficiency of their joint operation is largely determined by the conformance degree and the speed of the two-way information exchange.
The developed system provided a significant operator's comfort increase by providing him with a single user interface to manage various technological units from one operator station and an additional volume of information and services.

\section{REFERENCES}

1. Ostroukh, A.V. (2013). Systems of Artificial Intelligence in the Industry, the Robotics and the Transport Complex. Krasnoyarsk, RU: PublishingHouseScienceandlnnovationCenter, Ltd.

2. Ostroukh, A.V. \&Surkova N.E. (2015). Intelligence Information Systems and Technologies.Krasnoyarsk, RU: Publishing House Science and Innovation Center, Ltd.

3. Ostroukh, A.V. \& Tian, Yu. (2014). Automated system for monitoring production-technological and organizational-economic activity of industrial enterprises. Instruments and Systems: Monitoring, Control, and Diagnostics, (3), 14-21.

4. Tian, Yu., Nguen, D.T., Chaudhary, R.R. \&Ostroukh, A.V. (2014). Automated monitoring production technological and organizational - economic activities of an industrial enterprise. Automation and Control in Technical Systems, 3(1.2), 16-31. DOI: 10.12731/2306-1561-2014-1-16.

5. Ostroukh, A.V. \& Tian, Yu. (2013). Integration of monitoring system components. Young Scientist, 6(10), 182-185.

6. Ostroukh, A.V. \& Tian, Yu. (2014). Development of the information and analytical monitoring system of technological processes of the automobile industry enterprise. In the World of Scientific Discoveries, Series $\mathrm{B}, 2(1), 92-102$.

7. Ostroukh, A.V.,Gimadetdinov, M.K. \& Popov, V.P. (2015). Selection Process Equipment for Automated Crushing Plant.Automation and Control in Technical Systems,4(2), 35-45. DOI: 10.12731/2306-15612015-2-4.

8. Gimadetdinov, M.K. \&Ostroukh, A.V. (2014). List and Sequence of Solutions for Automated Crushing and Screening Production. Automation and Control in Technical Systems, 3(4), 55-61. DOI: 10.12731/2306-1561-2014-4-6.

9. Vorobeva, A.V., Ostroukh, A.V. Gimadetdinov, M.K., Wai Phyo Aung \&Myo Lin Aung. (2015). Development of mathematical models and methods of optimal control of automated crushing and screening process. IndustrialAutomaticControlSystemsandControllers, (1), 9-16.

10. Ostroukh, A.V.,Gimadetdinov, M.K., Borshch V.V.\&Vorobeva, A.V. (2014). Development statistical modeling algorithm optimal control automated crushing and screening process. Industrial Automatic Control Systems and Controllers, (12), 3-10. 
11. Gimadetdinov, M.K., Popov, V.P. \&Ostroukh, A.V. (2015). Automation of Crushing and Screening Production with a Full Range of Technological Operations and Related Processes. Industrial Automatic Control Systems and Controllers, (9), 10-18.

12. Ostroukh, A.V., Vasiliev, Yu. E. \&Kotlyarsky, E.V. (2015). Automated Control System Milling Unit for the Mineral Powders Plant. IndustrialAutomaticControlSystemsandControllers, (10), 3-10.

13. Ostroukh, A. V., Nedoseko, I. V., Surkova, N. E., \&Bulatov, B. G. (2016). Automated Control System for the Milling Unit of Mineral Powders Plant. International Journal of Applied Engineering Research, 11(4), 2625-2628.

14. Ostroukh, A., Maksimychev, O., Nikolaev, A., Kolbasin, A., \&Nedoseko, I. (2016). Development of automation of the drying and milling unit for the mineral powders plant. ARPN JournalofEngineeringandAppliedSciences, 11(9), 5717-5721.
15. Salniy, A.G., Kukharenko, V.N., Nikolaev, A.B.\&Ostroukh, A.V. (2013). General Principles of SCADA Design. AutomationandControlinTechnicalSystems, 2(2), 8-12.

16. Varlamov, O.O. (2002). Evolutionary databases and knowledge for adaptive synthesis of intelligent systems. MIVAR Information Space. Moscow, RU: Radio and Communication, Ltd.

17. Varlamov, O.O. (2011). MIVAR: Transition from Productions to Bipartite Graphs MIVAR Nets and Practical Realization of Automated Constructor of Algorithms Handling More than Three Million Production Rules.https://arxiv.org/abs/1111.1321

18. Varlamov,O.O. (2015). Mivar Role and Place in Computer Sciences, Artificial Intelligence Systems and Informatics.Radio Industry,(3), 10-27.

19. Shadrin, S.S., Varlamov, O.O. \& Ivanov, A.M. (2017). Experimental Autonomous Road Vehicle with Logical Artificial Intelligence. Journal of Advanced Transportation. DOI:10.1155/2017/2492765. 